\title{
Modeling Elasto-Plastic Behavior of Polycrystalline \\ Grain Structure of Steels at Mesoscopic Level
}

\author{
Marko Kovač ${ }^{1, *}$, Leon Cizelj $^{1}$ \\ ${ }^{1}$ Jožef Stefan Institute, Reactor Engineering Division, Ljubljana, Slovenia
}

\begin{abstract}
The multiscale model is proposed to explicitly account for the inhomogeneous structure of polycrystalline materials. Grains and grain boundaries are modeled explicitly using Voronoi tessellation. The constitutive model of crystal grains utilizes anisotropic elasticity and crystal plasticity. Commercially available finite element code is applied to solve the boundary value problem defined at the macroscopic scale. No assumption regarding the distribution of the mesoscopic strain and stress fields is used, apart the finite element discretization. The proposed model is then used to estimate the minimum size of polycrystalline aggregate of selected reactor pressure vessel steel (22 NiMoCr 37 ), above which it can be considered macroscopically homogeneous. Elastic and rate independent plastic deformation modes are considered. The results are validated by the experimental and simulation results from the literature.
\end{abstract}

\section{KEY WORDS}

Polycrystalline material, elasto-plastic material behavior, mesoscale, Voronoi tessellation, finite elements, crystal plasticity

\section{INTRODUCTION}

During a severe accident the pressure boundary of reactor coolant system can be subjected to extreme loads, which might cause its failure. Reliable estimation of extreme deformations can be crucial to predict the course of events and estimate the potential consequences of severe accident. Conventional structural mechanics has been traditionally applied to model and predict the response of materials and

\footnotetext{
* Corresponding author. Address; Jožef Stefan Institute, Reactor Engineering Division, Jamova 39, 1000 Ljubljana,Slovenia, email:marko.kovac@ijs.si.
} 
structures. However, the models of inelastic deformation are size and scale independent. In contrast, there is considerable experimental evidence that plastic flow in crystalline solids is inherently size dependent over a wide range of size scales. It is over the mesoscale size range - scale of grains in polycrystalline materials - that key deformation and fracture processes in a variety of structural materials take place (Needleman, 2000).

One of the most important drawbacks of conventional structural mechanics remains the idealization of inhomogeneous structure of materials (Nemat-Nasser and Hori, 1993). It might therefore fail to predict the material behavior, when the inhomogeneities start to dominate its response. For large structures (compared to the size of inhomogeneities), these effects typically become dominant while approaching limit loads. However, for relatively small structures, the effects of inhomogeneities may become noticeable already at the level of normal service loads (Kröner, 1986b; Needleman, 2000).

A variety of approaches, which tried to predict the effective overall behavior of the polycrystalline aggregate from a known behavior of the monocrystal, developed over the years. These include the earliest approaches by Voigt (Voigt, 1889) and Reuss (Reuss, 1929), who did not consider any particular grain shape. Other more recent examples of simplified grain geometries - with increasingly sophisticated overall treatment of the problem - include squares ( $\mathrm{Kad}$ et al., 1995), cubes (Frank et al., 2003), and Wigner-Seitz cells (Beaudoin et al., 2000). These approaches provided reasonable estimates of the effective overall behavior of the polycrystals, however they paid little attention to the details of the mesoscopic behavior.

Recent fast development of computers enabled expansion of models with increasingly realistic treatment of mesoscopic features, including the shapes of the grains. Models, which use stochastic methods such as Voronoi tessellation to accommodate the grain structure, were introduced only recently (for review, see for example (Beaudoin et al., 1995), (Barbe et al., 2001), and (Cailletaud et al., 2003)). The most sophisticated models applied explicit modeling of the grain boundaries by fitting the computational cells into the Voronoi tessellation (e.g., (Ghosh et al., 1995; Weyer, 2001; Kanit et al., 2003)). This increases the potential to predict the local 
deformations including for example shear banding and also provides the framework to simulate local damage mechanisms. However, these models typically concentrated on a few selected mesoscopic features and simplified or neglected others.

Crystal plasticity, which assumes that the crystalline slip is a predominant deformation mechanism of monocrystal, is typically implemented in these models to describe inelastic material behavior of the basic constituents (e.g., monocrystals). Finite element method is used as a standard tool for obtaining sub-grain stress and strain fields (Needleman, 2000; Cailletaud et al., 2003).

A generalized multiscale model of polycrystalline aggregate, which overcomes the a priori assumptions applied by previous approaches, is therefore proposed. The most distinctive features of the proposed multiscale model, which offers minimization of the a priori assumptions applied by previous approaches are:

(1) Explicit modeling of grains and grain boundaries using the Voronoi tessellation, which allows explicit account of incompatibility strains at the grain boundaries, and offers a significant potential to utilize specific models of grain boundaries, including intergranular damage, in the future.

(2) Defining and solving the boundary value problem at the macroscopic level with commercially available finite element solver.

(3) No a priori assumptions on the sub-grain stress and strain field distribution are used, apart from the finite element discretization.

The analysis is limited to 2-D structures due to the high computational efforts. The proposed model is however easily extendable to 3-D.

In the numerical examples, the proposed model is used to estimate the minimum size of polycrystalline aggregate above which it can be considered macroscopically homogeneous. This can be used as an orientation value to predict the lower bound of domain of the conventional structural mechanics. The material properties were selected to mimic the behavior of the German reactor pressure vessel 22 NiMoCr 37. This analysis was performed as a part of the LISSAC (Limit Strains for Severe Accident Conditions) project (Krieg and Seidenfuß, 2003; Cizelj et al., 2002), which among others tried to experimentally determine the size effect in inelastic 
deformations by exploring a series of geometrically similar tensile specimens with sizes ranging from 4 to $400 \mathrm{~mm}$. The predicted RVE sizes are confirmed by the experimental results of the LISSAC project and by comparison with computational results published by (Nygards, 2003).

In addition, the potential of the proposed model for applications in damage processes involving intergranular cracking was explored and reported elsewhere (Cizelj and Riesch-Oppermann, 2002; Cizelj and Kovač, 2003). The proposed model was also used to predict the overall properties and anisotropy of small polycrystalline aggregates (smaller then the representative volume element) (Kovač, 2004) and estimation of correlation length (Simonovski et al., 2004; Simonovski et al., Submitted).

\section{THEORETICAL BACKGROUND}

The proposed model of polycrystalline aggregate can be essentially divided into modeling the random grain structure, calculation of strain/stress field and obtaining overall properties of the aggregate. Basic features are:

- The random polycrystalline structure is represented by a Voronoi tessellation.

- The constitutive model of randomly orientated crystal grains (monocrystals) assumes anisotropic elasticity and crystal plasticity. The latter assumes that plastic deformation is caused by crystalline slip on predefined slip planes of crystal lattice. Slip planes and directions are defined by random orientation of crystal lattice. Finite element method is used to obtain numerical solutions of strain and stress fields.

- The overall properties of the polycrystalline aggregate are obtained by homogenization procedure.

- The representative volume element is estimated by comparison of the overall properties of polycrystals produced by complementary set of macroscopic boundary conditions.

\subsection{Voronoi Tessellation}

The concept of Voronoi tessellation has recently been extensively used in the materials science, especially for modeling random microstructures like aggregates of 
grains in polycrystals (Riesch-Oppermann, 1999; Weyer et al., 2002; Nygards, 2003), patterns of intergranular cracks (Cizelj and Riesch-Oppermann, 2002), and composites (Johansson, 1995). A Voronoi tessellation represents a cell structure constructed from a Poisson point process by introducing planar cell walls perpendicular to lines connecting neighboring points. This results in a set of convex polygons/polyhedra (Figure 1) embedding the points and their domains of attraction, which completely fill up the underlying space. All Voronoi tessellations used for the purpose of this paper were generated by the code VorTess (Riesch-Oppermann, 1999).

Discretization of the Voronoi polygons into triangular finite elements is straightforward. Unfortunately, the numerical quality of triangular finite elements is generally poor. Planar quadrilateral elements were therefore used in this paper. One of the basic requirements for reliable finite element analysis is suitable shape of the finite elements in the mesh. The reliability of analysis can be improved, if only "meshable" tessellations are taken into account. Use of "meshable" tessellations poses limitations to tolerable distortion from ideally square shape of finite elements, which cause that only a subset of all possible tessellations is used in the analysis. Such bias is considered to be small compared to the error caused by the 2-D approximation of grain structure (Weyer, 2001). Further details on "meshable" tessellations and automatic meshing algorithms are employed in this paper given in (Weyer et al., 2002).

\subsection{Constitutive Model of Monocrystal}

The main features of the elasto-plastic constitutive model of monocrystal are briefly explained below.

Each crystal grain in the polycrystalline aggregate is assumed to behave as an anisotropic continuum (Nye, 1985). Random orientation of crystal lattice differs form grain to grain. Constitutive relations in linear elasticity are given by the generalized Hooke's law:

$$
\sigma_{i j}=C_{i j k l} \varepsilon_{k l},
$$


where $\sigma_{i j}$ represents the second rank stress tensor, $C_{i j k l}$ represents the fourth rank stiffness tensor and $\varepsilon_{i j}$ represents the second rank strain tensor. Indices $i, j, k$ and $l$ are running from 1 to 3 . The inverse of the stiffness tensor is called compliance tensor $D_{i j k l}$ and is defined as:

$$
\varepsilon_{i j}=D_{i j k l} \cdot \sigma_{k l}
$$

Crystal plasticity used in the proposed model follows the pioneering work of Taylor (Taylor, 1938), Hill and Rice (Hill and Rice, 1972) and Asaro (Asaro, 1983). It is assumed that the plastic deformation is a result of crystalline slip only and the crystalline slip is driven by resolved shear stress $\tau^{(\alpha)}$ (Asaro, 1983; Huang, 1991):

$$
\tau^{(\alpha)}=m_{i}^{(\alpha)} \cdot \sigma_{i j} \cdot s_{j}^{(\alpha)}
$$

where $\alpha$-th slip system is defined by a combination of slip plane (determined by normal $m_{i}^{(\alpha)}$ ) and slip direction $\left(s_{j}^{(\alpha)}\right)$. The number of slip systems and their orientations depend on the crystal lattice. Stress rate can be defined as:

$$
\dot{\sigma}_{i j}=C_{i j k l} \cdot\left(\dot{\varepsilon}_{k l}-\dot{\varepsilon}_{k l}^{p}\right)=C_{i j k l} \cdot\left(\dot{\varepsilon}_{k l}-\sum_{\alpha} \frac{1}{2} \dot{\gamma}^{(\alpha)}\left(s_{i}^{(\alpha)} m_{j}^{(\alpha)}+s_{j}^{(\alpha)} m_{i}^{(\alpha)}\right)\right),
$$

where $\dot{\sigma}_{i j}$ is the stress rate tensor, $\dot{\mathcal{E}}_{k l}$ is the strain rate tensor, $\dot{\mathcal{\varepsilon}}_{k l}^{p}$ is the plastic strain rate tensor and $\dot{\gamma}^{(\alpha)}$ is the slipping rate of the $\alpha$-th slip system. The slipping rate $\dot{\gamma}^{(\alpha)}$ is assumed to be governed by the resolved shear stress $\tau^{(\alpha)}$ in a visco-plastic framework (Huang, 1991):

$$
\dot{\gamma}^{(\alpha)}=\dot{a}^{(\alpha)}\left(\frac{\tau^{(\alpha)}}{g^{(\alpha)}}\right)\left(\left|\frac{\tau^{(\alpha)}}{g^{(\alpha)}}\right|\right)^{n-1}
$$

where $\dot{a}^{(\alpha)}$ is reference strain rate, $n$ the strain rate sensitivity parameter and $g^{(\alpha)}$ the current strain hardened state of the crystal. In the limit as $n$ approaches infinity this power law approaches that of a rate-independent material. The current strain hardened state $g^{(\alpha)}$ is defined by:

$$
\dot{g}^{(\alpha)}=\sum_{\beta} h_{\alpha \beta} \dot{\gamma}^{(\beta)}
$$


where $h_{\alpha \beta}$ are the slip hardening moduli. Different proposals of hardening moduli could be found in literature (e.g., (Asaro, 1983; Bassani and Wu, 1991)), all of them relying on empirical models. Peirce et al. (Peirce et al., 1982) and Asaro (Asaro, 1983) hardening law is used in numerical example. Self- $\left(h_{\alpha \alpha}\right)$ and latent-hardening moduli $\left(h_{\alpha \beta}\right)$ are defined as:

$$
h_{\alpha \alpha}=h(\gamma)=h_{0} \operatorname{sech}^{2}\left|\frac{h_{0} \gamma}{\tau_{S}-\tau_{0}}\right|, \quad h_{\alpha \beta}=q h(\gamma),(\alpha \neq \beta),
$$

where $h_{0}$ is the initial hardening modulus, $\tau_{0}$ the yield stress, which equals the initial value of current strength $g^{(\alpha)}(0), \tau_{S}$ the break-through stress where large plastic flow initiates, $\gamma$ the cumulative slip and $q$ is hardening factor.

A user subroutine (Huang, 1991), which incorporates anisotropic elasticity and crystal plasticity with finite-strain and finite-rotation formulations, was used in the commercially available finite element code ABAQUS/Standard (ABAQUS/Standard, 2002).

\subsection{Overall Properties of the Polycrystalline Aggregate}

The boundary value problem is defined and solved at the macroscopic level. However, explicit modeling of the grain structure including grain boundaries causes that the main results of the proposed model are the mesoscopic strain and stress fields. To obtain the overall macroscopic properties of the polycrystalline aggregate, appropriate homogenization of the mesoscopic fields is necessary. The following algorithm has been used:

- The mesoscopic stress $\sigma_{i j}^{L}$ and strain $\varepsilon_{i j}^{L}$ tensors in each integration point are rotated from the local to the global coordinate system:

$$
\sigma_{i j}^{G}=\sigma_{i j}^{L} \cdot Q_{i k} \cdot Q_{j l}, \quad \varepsilon_{i j}^{G}=\varepsilon_{i j}^{L} \cdot Q_{i k} \cdot Q_{j l},
$$

where $\sigma_{i j}^{G}$ and $\varepsilon_{i j}^{G}$ represents stress and strain tensor, respectively, in the global coordinate system and $Q_{i j}$ represents rotation tensor from the local to the global coordinate system. Current rotation of the material in the specific integration point depends on initial random orientation and the change due to the finite rotation formulation. 
- The macroscopic stress $\left\langle\sigma_{i j}\right\rangle$ and strain $\left\langle\varepsilon_{i j}\right\rangle$ tensors are obtained by averaging the mesoscopic stress and strain tensors in the global coordinate system over the volume of the polycrystalline aggregate $V$ :

$$
\left\langle\sigma_{i j}\right\rangle=\frac{1}{V} \int_{V} \sigma_{i j}^{G} d V, \quad\left\langle\varepsilon_{i j}\right\rangle=\frac{1}{V} \int_{V} \varepsilon_{i j}^{G} d V .
$$

- The equivalent macroscopic stress and strain are then calculated from the macroscopic stress and strain tensors to make results comparable with uniaxial tensile tests.

\subsection{Estimation of Representative Volume Element Size}

Geometrically similar components, which are larger than the representative volume element (RVE), will all have the same macroscopic response, regardless of their size and their inhomogeneity (Nemat-Nasser and Hori, 1993). However, this is not the case with components smaller than RVE, where microstructure might play an important role on the macroscopic response. RVE is therefore defined as the minimum size of the polycrystalline aggregate above which the influence of grain structure of the selected material on the macroscopic response is negligible. From the computational viewpoint, the RVE size is defined as equivalence of stiffness $C^{*}{ }_{i j k l}$ and inverse compliance $D^{*}{ }_{i j k l}$ tensors (Kröner, 1986a):

$$
C_{i j k l}^{*} \cong\left(D_{i j k l}^{*}\right)^{-1} \text {. }
$$

Equation (10) is in general not valid for the polycrystalline aggregates smaller than RVE. As a consequence, analysis of parts smaller than RVE with the methods of conventional structural mechanics might not yield meaningful results.

The different behavior of both tensors is governed by the size of the aggregate and the macroscopic boundary conditions (Nemat-Nasser and Hori, 1993). The macroscopic stress $\left\langle\sigma_{i j}\right\rangle$ and strain tensors $\left\langle\varepsilon_{k l}\right\rangle$ are completely defined by the properties of and interaction between the monocrystals and may be used to estimate the macroscopic stiffness $C_{i j k l}^{*}$ or macroscopic compliance tensor $D_{i j k l}^{*}$ : 


$$
\left\langle\sigma_{i j}\right\rangle=C^{*}{ }_{i j k l}\left\langle\varepsilon_{k l}\right\rangle, \quad\left\langle\varepsilon_{k l}\right\rangle=D^{*}{ }_{i j k l}\left\langle\sigma_{i j}\right\rangle .
$$

It is useful to note here that the macroscopic stiffness tensor (eq. (11a)) assumes stress driven macroscopic boundary conditions, while the macroscopic compliance tensor (eq. (11b)) assumes displacement driven macroscopic boundary conditions, Figure 2.

For elastic and rate independent inelastic materials with nearly linear stress strain relationship, equation (10) can be reasonably approximated by:

$$
\left\langle\sigma_{e q_{s}}\right\rangle \cong\left\langle\sigma_{e q_{d}}\right\rangle
$$

where $\left\langle\sigma_{e q}\right\rangle$ denotes the macroscopic equivalent stresses and indexes ${ }_{s}$ and ${ }_{d}$ denote stress and displacement boundary conditions, respectively.

The size of the RVE can be extrapolated from the known behavior of a set of polycrystalline aggregates, which are smaller than RVE (Weyer, 2001). Relation between the macroscopic stiffness and compliance tensors for polycrystalline aggregates smaller than RVE can be written as (Weyer, 2001):

$$
C_{i j k l}^{*} \cdot D_{k l m n}^{*}=I_{i j m n}+O\left(V / V_{R V E}\right),
$$

where $V_{R V E}$ represents the RVE size, $V$ size of the polycrystalline aggregate, $I_{i j m n}$ 4-th rank unit tensor and $O$ a residuum. Assuming proportionality between number of grains in the polycrystalline aggregate and its size and validity of eq. (12), one can use (Kovač and Cizelj, 2002):

$$
\frac{\left\langle\sigma_{e q_{s}}\right\rangle}{\left\langle\sigma_{e q_{d}}\right\rangle}=1+O\left(i / i_{R V E}\right),
$$

where $i_{R V E}$ represents the number of grains in RVE and $i$ the number of grains in the polycrystalline aggregate smaller than RVE. It is customary to assume that RVE is achieved when residuum $O$ is smaller than $1 \%$ (Kanit et al., 2003). 
For polycrystalline aggregates with periodic boundary conditions, Nygards (Nygards, 2003) suggested a measure for the RVE, which is based on the scatter caused by initial random crystal lattice orientations:

$$
O_{S}=\frac{S\left(\sigma_{e q}\right)}{\sigma_{\text {eq ave }}},
$$

where $S\left(\sigma_{e q}\right)$ is standard deviation of equivalent stress variability due to the crystal lattice orientation, $\sigma_{e q}$ ave is average equivalent stress (obtained from a large enough polycrystalline aggregate, e.g., 500-grains aggregate), and $O_{S}$ is residuum. The RVE is achieved at $O_{S}<O_{S t h}$, with typical choice of $O_{S t h}$ being again about $1 \%$.

\section{NUMERICAL EXAMPLE AND DISCUSSION}

In the numerical example, the performance of the proposed model is demonstrated. First, the mesoscopic fields are shown. Then, the number of grains in the aggregate for which the effect of the initial orientations and the macroscopic boundary conditions becomes negligible for the macroscopic behavior (i.e., the representative volume element, RVE) is estimated. The predicted RVE sizes are confirmed by the experimental results of the LISSAC project and by comparison with computational results published by (Nygards, 2003).

\subsection{Input Data}

A set of Voronoi tessellations with 14, 23, 53, 110 and 212 grains was used in the analysis to simulate the polycrystalline aggregates with respective sizes $0.1 \mathrm{~mm} \times$ $0.07 \mathrm{~mm}, 0.13 \mathrm{~mm} \times 0.09 \mathrm{~mm}, 0.2 \mathrm{~mm} \times 0.14 \mathrm{~mm}, 0.28 \mathrm{~mm} \times 0.2 \mathrm{~mm}$, and $0.4 \mathrm{~mm}$ $\times 0.28 \mathrm{~mm}$. The average grain size of $0.023 \mathrm{~mm}$ was assumed, as reported within the LISSAC project (Materna-Morris et al., 2002). Only one realization of tessellation per selected size was analyzed. Each tessellation was simulated with 30 different random orientations of crystal lattices, each of them with two complementary stress and displacement boundary conditions (Figure 2). This is consistent with observation that the variability due to grain shapes is relatively small compared to the variability due to grain orientations (Weyer, 2001). 
The pressure vessel steel 22 NiMoCr 37 with bainitic structure and body-centered cubic (b.c.c.) crystals is simulated in the numerical example. Three families of slip planes: $\{110\},\{112\}$, and $\{123\}$ and one family of slip directions: $<111>$ were modeled within the b.c.c. crystal lattice (Nemat-Nasser et al., 1998). Elasticity properties of the selected material are obtained from the literature for $\alpha$-Fe with b.c.c. crystal lattice (Nye, 1985; Grimvall, 1999). It is assumed that small amounts of alloying elements do not change the elastic properties of a crystal grain significantly. The nonzero components of the stiffness tensor (eq. (1)) are therefore taken as $C_{i i i i}=230 \mathrm{GPa}, C_{i j i j}=135 \mathrm{GPa}$ and $C_{i j i j}=117 \mathrm{GPa}$. Material parameters for plasticity were selected as follows (Julisch, 2002). The strain rate sensitivity parameter $n=50$ and the reference strain rate $\dot{a}^{(\alpha)}=0.001 \mathrm{~s}^{-1}$ (eq. (5)) were selected to result in strain rate independent behavior (Nemat-Nasser et al., 1998). The initial hardening modulus $h_{0}=70 \mathrm{MPa}$, the break-through stress $\tau_{S}=15.5 \mathrm{MPa}$, yield stress $\tau_{0}=155 \mathrm{MPa}$ and hardening factor $q=1$ (eq. (7)) were optimized to comply with a series of low velocity tensile tests performed at room temperature within the LISSAC project (Kieselbach, 2001).

\subsection{Mesoscopic Strain/stress Fields}

The mesoscopic stress and strain fields represent the direct result of the proposed model. Typical examples of mesoscopic fields obtained are shown in Figure 3 left (equivalent Von Mises stress) and Figure 3 right (equivalent strain). The aggregate with 212-grains and macroscopic displacement boundary conditions is shown, loaded biaxially to $p_{1}=1155 \mathrm{MPa}$ and $p_{2}=578 \mathrm{MPa}$ (depicted with circle in Figure 4). This particular loading condition was selected since it clearly illustrates the extremely inhomogeneous nature of the mesoscopic fields: while the macroscopic equivalent stress $\left(\left\langle\sigma_{e q}\right\rangle=518 \mathrm{MPa}\right)$ exceeded the macroscopic yield strength $\left(\sigma_{Y}=440 \mathrm{MPa}\right)$ significantly leading to the macroscopic equivalent strain of about $6 \%$, there is still a considerable fraction of grains where none or negligible slipping has taken place.

The local mesoscopic equivalent stresses depicted in Figure 3 left are up to $60 \%$ higher than the macroscopic (average) equivalent stress. The stress field also shows high gradients at the grain boundaries, which are mainly caused by the incompatible deformation of neighboring grains. The plastic strain is concentrated within shear bands (Figure 3 right). The local equivalent strains are up to $500 \%$ higher than 
macroscopic equivalent strain. Shear bands typically develop at the grain boundaries (they are less pronounced when passing through the grains) in directions of about $50^{\circ}$ from x-axis. The typical distance between shear bands is in the order of the grain size. The characteristic appearance of mesoscopic features (e.g., stress gradients along the grain boundaries and shear bands) clearly highlights the advantages of the explicit modeling of the grains and grain boundaries.

\subsection{Estimation of RVE Size}

The representative volume element (RVE) is defined as the minimum number of grains in the aggregate, for which the effect of the initial orientations and the macroscopic boundary conditions become negligible for the macroscopic behavior. In general, the RVE is expected to depend on the stress-strain relationship. The nearly bi-linear behavior of the stress-strain relation (as for example depicted in Figure 4) suggests that for this particular material it is sufficient to estimate the RVE at two distinct points, selected as follows.

- For elastic deformation mode at biaxial remote load of $p_{1}=200 \mathrm{MPa}$ and $p_{2}=100 \mathrm{MPa}$ (Figure 2).

- For plastic deformation mode at biaxial remote load $p_{1}=1094 \mathrm{MPa}$ and $p_{2}=547 \mathrm{MPa}$.

The size of the representative volume element size was extrapolated from the results obtained for aggregates with 14, 23, 53, 110 and 212 grains, each of them analyzed for 30 different random orientations of crystal lattices and two complementary boundary conditions.

The macroscopic equivalent stresses and strains at given fixed remote load for all analyzed aggregates are shown in Figure 5. The values obtained for elastic deformation mode are shown on the left and the ones for plastic deformation mode on the right. $d$ and $s$ in the legend refer to displacement and stress boundary conditions, respectively. ave refers to values averaged over 30 different randomly orientated crystal lattices for each boundary condition. The numbers following abbreviation denote number of grains in the respective aggregate. The trends of 
averaged values for displacement and stress boundary conditions are depicted with thick arrows, marked with trend $d$ and trend $s$, respectively.

Two effects appear with increasing number of grains: (1) decrease of scatter and (2) clear trend of average values towards each other. For elastic deformation mode (Figure 5 left) the common average $\left(\left\langle\varepsilon_{e q}\right\rangle=0.0515 \%\right.$ and $\left.\left\langle\sigma_{e q}\right\rangle=95.2 \mathrm{MPa}\right)$ coincide with the analytical solution for equivalent homogenous material with material parameters: $E=210 \mathrm{GPa}$ and $v=0.29$. The analytical solution for equivalent homogenous material for plastic deformation mode could unfortunately not be given. However, the expected value for equivalent homogenous material $\left(\left\langle\varepsilon_{e q}\right\rangle=5.0 \%\right.$ and $\left\langle\sigma_{e q}\right\rangle=503 \mathrm{MPa}$; Figure 5 right) was determined as point of intersection of trends of average values obtained by two complementary sets of boundary conditions.

It can be also noted that the displacement boundary conditions consistently cause stiffer response as compared to the stress boundary conditions. The scatter of results in Figure 5 right has increased tremendously as compared to Figure 5 left. This is caused by the extremely inhomogeneous mesoscopic fields, as illustrated in Figure 3. A considerable fraction of the aggregate could remain elastic even at macroscopic equivalent strains exceeding 5\%. The amount of elastic grains at given macroscopic equivalent strain varies considerably and is strongly influenced by the initial random orientations of the lattice.

In order to estimate the size of the RVE, it is useful to rearrange the data from Figure 5 in the following way: first, fix the macroscopic equivalent strain at $\left\langle\varepsilon_{e q}\right\rangle=0.0515 \%$ and $5.0 \%$ for elastic (left) and plastic deformation mode (right), respectively. Then, plot the average stresses obtained for 30 random lattice orientations as a function of number of grains in the aggregate for both boundary conditions (Figure 6). The scatter of stresses is depicted by the error bars placed at \pm one standard deviation. Finally, fit the average values with a third order polynomial (Weyer, 2001).

Figure 6 shows rather fast decrease of scatter due to the random orientations of lattice with increasing number of grains. Also, the clearly different behavior of results for aggregates with complementary boundary conditions tends to converge 
with increasing number of grains. It is worth noting that the averages and standard deviations of stresses plotted in Figure 6 left and Figure 6 right could also be used for example to predict the scatter of material properties for the continuum based analysis of components smaller than RVE. More details about predictions of material properties of small aggregates is available in (Kovač, 2004).

Fitted lines, extrapolated towards large number of grains, clearly tend to each other. The common average for the elastic deformation mode coincides with the anticipated analytical solution (Figure 6, left). The RVE is now estimated in accordance with eq. (14). Assuming the residuum $O$ of about $1 \%$, the RVE is to be at least 372 grains for elastic deformation mode and 763 grains for plastic deformation mode. This corresponds to the polycrystalline aggregates of $0.53 \mathrm{~mm} \times 0.38 \mathrm{~mm}$ (elastic) and $0.78 \mathrm{~mm} \times 0.55 \mathrm{~mm}$ in size (plastic).

\subsection{Validation of Results}

From discussion in Section 3.3 it clearly follows that the size of the RVE is closely linked to the scatter of the mesoscopic fields. This is consistent with the measure for the RVE (residuum $O_{S}$, eq. (15)) suggested for the polycrystalline aggregates with periodic boundary conditions by Nygards (Nygards, 2003). Nygards analyzed both 2$\mathrm{D}$ and 3-D aggregates and provided empirical relations between the residuum $O_{S}$ and number of grains for aggregates of various anisotropically elastic cubic metals, including iron, loaded by uniaxial and equibiaxial remote loads. It is therefore useful to compare the results of the proposed model with results obtained by Nygards.

Figure 7 summarizes the comparison of results obtained by the proposed model and by 2-D model of Nygards. Three sets of curves are plotted for three different types of loading. For uniaxial load, the curve proposed by Nygards for aggregate with periodic boundary conditions is clearly embedded by the results of the proposed model with complementary boundary conditions. Good agreement is also observed in the case of equibiaxial loading. Finally, all groups of curves clearly tend to a single curve with increasing number of grains. It is therefore reasonable to expect that the results of the proposed model for the 2:1 biaxial case are also correct. 
The RVE's deduced from Figure 7 with $O_{S}=1 \%$ are 102 grains for equibiaxial load, between 110 and 210 grains for the 2:1 biaxial load and 379 grains for the equibiaxial load. This differs considerably from the 372 grains for 2:1 biaxial load estimated in Section 3.3. This difference primarily reflects the importance of the macroscopic boundary conditions, especially when analyzing aggregates smaller than RVE.

Qualitative equivalence of 2-D and 3-D models of the grain structures is clearly shown in (Nygards, 2003). A notable difference however remains in the estimated size of the RVE. 3-D RVE estimates result in approximately twice as many grains per aggregate as 2-D estimates. This results in slight conservativity of the RVE sizes estimated with 2-D models.

Indirect confirmation of the estimated RVE for plastic deformation follows from the experimental results of the LISSAC project (Krieg and Seidenfuß, 2003). A series of tensile tests of the pressure vessel steel $22 \mathrm{NiMoCr} 37$ were executed with geometrically similar specimens of sizes between 4 and $400 \mathrm{~mm}$. While some moderate size effects were observed at large strains dominated by the development of ductile damage, no size effects were observed within the plastic deformation until strains of about $10 \%$. This is consistent with our estimate of the RVE for plastic deformation, which suggest macroscopically homogeneous plastic deformation in specimens exceeding roughly $1 \mathrm{~mm}$ in size.

\section{SUMMARY}

A novel multiscale model of elasto-plastic behavior of a polycrystalline aggregate was proposed in this paper. Its development was motivated by accounting for the most important features of the grain structure of polycrystalline materials, which is neglected by the conventional continuum mechanics. The Voronoi tessellation is used to explicitly model the random grain structure. The crystal grains are modeled as a randomly oriented monocrystals obeying continuum elasto-plastic constitutive models. Compatible displacements are assumed along the explicitly modeled grain boundaries, which gives rise to the incompatibility strains at the grain boundaries. The boundary value problem is defined and solved at the macrosopic scale using standard crystal plasticity models, commercially available finite element solver and 
two complementary sets of macroscopic boundary conditions. The basic reason for the current limitation to 2-D structures is very high computational intensity of the model.

The material properties used in the numerical examples have been selected to simulate the reactor pressure vessel steel $22 \mathrm{NiMoCr} 37$. Elastic and rate independent plastic deformation modes were considered. The mesoscopic stress and strain fields were found to be extremely heterogeneous. This includes development of high stress gradients along the grain boundaries and shear bands, both being consequences of explicit modeling of the grain boundaries.

The proposed model was then used to estimate the minimum size of polycrystalline aggregate, above which it can be considered macroscopically homogeneous (the representative volume element, RVE). This can be used as an orientation value to predict the lower bound of domain of conventional structural mechanics (below which it is not able to describe the polycrystalline material behavior accurately).

The RVE size was estimated from the differences of response caused by complementary boundary conditions. The displacement boundary conditions caused consistently stiffer response of the polycrystalline aggregate than the stress boundary conditions. The difference in response however rapidly diminished with the increasing number of grains in the aggregate and resulted in the estimate of the RVE. Due to the nearly bi-linear nature of stress-strain curves, the RVEs were estimated at two points, representative for elastic and plastic deformation modes.

Further validation of the model showed compliance with the series of tensile test of geometrically similar specimens of different sizes exceeding the estimated RVE, which showed no size effects for strains smaller than $10 \%$. Comparison with simulation results from the literature shoved very good agreement of scatter due to random orientations and qualitative equivalence of 2-D and 3-D models of the grain structures in elastic deformation modes. 
Additional advantages of the proposed model include flexibility of constitutive model describing monocrystal and a strong potential for modeling the intergranular damage processes. These will be part of the future work.

\section{ACKNOWLEDGEMENTS}

This work was supported by the EU within the project LISSAC (Limit Strains for Severe Accident Conditions, contract number FIKS-CT 1999-00012) and by Ministry of Education, Science and Sport of Republic of Slovenia with the grant for young researcher (contract number S2-106-002/18292/2000).

\section{REFERENCES}

Asaro, R. J. 1983. Crystal Plasticity. Journal of Applied Mechanics, 50, 921.

Barbe, F., Decker, L., Jeulin, D., and Cailletaud, G. 2001. Intergranular and intragranular behaviour of polycrystalline aggregates. Part 1:F.E. model. International Journal of Plasticity, 17, 513-536.

Bassani, J. L. and Wu, T. Y. 1991. Latent hardening in single crystals, II. Analytical characterization and prediction. Proceedings of the Royal Society of London. Series A, 435, 21-41.

Beaudoin, A. J., Acharya, A., Chen, S. R., Korzekwa, D. A., and Stout, M. G. 2000. Consideration of grain-size effect and kinetics in the plastic deformation of metal polycrystals. Acta Materialia, 48 (13), 3409-3423.

Beaudoin, A. J., Dawson, P. R., Mathur, K. K., and Kocks, U. F. 1995. A Hybrid Finite Element Formulation for Polycrystal Plasticity With Consideration of Macrostructural and Microstructural Linking. International Journal of Plasticity, 11 (5), 501-521.

Cailletaud, G., Forest, S., Jeulin, D., Feyel, F., Galliet, I., Mounoury, V., and Quilici, S. 2003. Some elements of microstructural mechanics. Computational Materials Science, 27 (3), 351-374.

Cizelj, L. and Kovač, M. 2003. Numerically simulated growth of short intergranulated cracks in elastoplastic polycrystalline aggregate.11th International Conference on Nuclear Engineering, The Japan Society of Mechanical Engineering, 36351-1-36351-7.

Cizelj, Leon, Kovač, Marko, Simonovski, Igor, Petrič, Zoran, Fabjan, Ljubo, and Mavko, Borut. 2002. Elastic-Plastic Behavior of Polycrystalline Aggregate with Stochastic Arrangement of Grains. Project LISSAC: Final Report. Rev 0. IJS-DP-8667, Jožef Stefan Institute, Ljubljana, Slovenia.

Cizelj, L. and Riesch-Oppermann, H. 2002. Modeling the Early Development of Secondary Side Stress Corrosion Cracks in Steam Generator Tubes Using Incomplete Random Tessellation. Nuclear Engineering and Design, 212 (1-3), 21-29.

Frank, G. J., Olson, S. E., and Brockman, R. A. 2003. Numerical models of orthotropic and lamellar grain structures. Intermetallics, 11 (4), 331-340. 
Ghosh, S., Lee, K., and Moorthy, S. 1995. Multiple scale analysis of heterogeneous elastic structures using homogenization theory and voronoi cell finite element method. Journal of Solids and Structures, 32 (1), 27-62.

Grimvall, G. 1999. Thermophysical Properties of Materials, North-Holland, Amsterdam.

Hibbit, Karlsson \& Sorensen Inc. 2002. ABAQUS/Standard. 6.3 Pawtucket, R.I., USA

Hill, R. and Rice, J. R. 1972. Constitutive analysis of elastic-plastic crystals at arbitrary strain. Journal of the Mechanics and Physics of Solids, 20 (6), 401-413.

Huang, Yonggang. 1991. A User-material Subroutine Incorporating Single Crystal Plasticity in the ABAQUS Finite Element Program. MECH-178, Harvard University, Cambridge, Massachusetts.

Johansson, T. 1995. Analytische Beschreibung von Experimenten an fäserverstärkten Keramiken zur Bestimmung von Grenzflächenparametern, VDI Verlag, Düsseldorf, Germany.

Julisch, Peter. 2002. Results of Static Tensile Tests with Large Specimens. MPA Stuttgart, Stuttgart.

Kad, B. K., Dao, M., and Asaro, R. J. 1995. Numerical simulations of stress-strain behavior in twophase $\alpha_{2}+\gamma$ lamellar TiAl alloy. Materials Science and Engineering A, 192-193, 97-103.

Kanit, T., Forest, S., Galliet, I., Mounoury, and Jeulin, D. 2003. Determination of the size of the representative volume element for random composites: statistical and numerical approach. International Journal of Solids and Structures, 40 (13-14), 3647-3679.

Kieselbach, R. 2001. Tensile Tests, Specimens $5 \mathrm{~mm}$ for LISSAC. Test Report, Nr. 201951/01, EMPA.

Kovač, M. 2004. Influence of Microstructure on Development of Large Deformations in Reactor Pressure Vessel Steel. University of Ljubljana.

Kovač, M. and Cizelj, L. 2002. The effect of grain structure on the elastic-plastic response of a polycrystalline aggregate. Strojniški vestnik, 48 (11), 580-590.

Krieg, R and Seidenfuß, M. 2003. Limit Strains for Severe Accident Conditions, Final Report of the EU-project LISSAC, Contract No. FIKS-CT1999-00012. FZKA 6854, Forschungszentrum Karlsruhe, Karlsruhe.

Kröner, E. 1986a. The Statistical Basis of Polycrystal Plasticity. In: J. Gittus, J. Zarka, and S. NematNasser, eds., Large Deformations of Solids: Physical Basis and Mathematical Modelling, Elsevier Applied Science, 27-40.

Kröner, E. 1986b. Statistical Modelling. In: J. Gittus and J. Zarka, eds., Modelling Small Deformations of Polycrystals, Elsevier Applied Science, 229-291.

Materna-Morris, E., Graf, P., and Zimmermann, H. 2002. Structure and Fracture of 22NiMoCr3 7. Forschungszentrum Karlsruhe, Karlsruhe.

Needleman, A. 2000. Computational Mechanics at the Mesoscale. Acta Materialia, 48, 105-124.

Nemat-Nasser, S. and Hori, M. 1993. Micromechanics: Overall Properties of Heterogeneous Materials, North-Holland, Amsterdam. 
Nemat-Nasser, S., Okinaka, T., and Ni, L. 1998. A Physically-based Constitutive Model for BCC Crystals with Application to Polycrystalline Tantalum. Journal of the Mechanics and Physics of Solids, 46 (6), 1009-1038.

Nye, J. F. 1985. Physical Properties of Crystals, Clarendon Press, Oxford.

Nygards, M. 2003. Number of grains necessary to homogenize elastic materials with cubic symmetry. Mechanics of Materials, 35 (11), 1049-1059.

Peirce, D., Asaro, R. J., and Needleman, A. 1982. Material Rate Dependence and Localized Deformation in Crystalline Solids. Acta metallurgica, 31, 1951.

Reuss, A. 1929. Berechnung der Fließgrenze von Mischkristallen auf grund der Plastizitätsbedingung für Einkristalle. Zeitung für angewandte Mathematik und Mechanik, 9, 49-58.

Riesch-Oppermann, Heinz. 1999. VorTess, Generation of 2-D Random Poisson-Voronoi Mosaics as Framework for the Micromechanical Modelling of Polycristalline Materials. Report FZKA 6325, Forschungszentrum Karlsruhe, Karlsruhe, Germany.

Simonovski, I., Kovač, M., and Cizelj, L. 2004. Estimating the correlation length of inhomogeneities in a polycrystalline material. Material Science and Engineering A.

Simonovski, I., Kovač, M., and Cizelj, L. Submitted. Correlation Length Estimation Issues In Stochastic Material Model. Nuclear Engineering And Design.

Taylor, G. I. 1938. Plastic strain in metals. Journal of Institute of Metals, 62, 307-324.

Voigt, W. 1889. Über die Beziehung zwischen den beiden Elasticitätskonstanten isotroper Körper. Wied. Ann., 38, 573-587.

Weyer, S. 2001. Experimentelle Untersuchung und mikromechanische Modellierung des Schädigungsverhaltens von Aluminiumoxid unter Druckbeanspruchung. Universität Karlsruhe.

Weyer, S., Fröhlich, A., Riesch-Oppermann, H., Cizelj, L., and Kovač, M. 2002. Automatic Finite Element Meshing of Planar Voronoi Tessellations. Engineering Fracture Mechanics, 69 (8), 945-958. 


\section{LIST OF FIGURES}

Figure 1: Voronoi tessellation with highlighted orientations of crystal lattices and finite element mesh

Figure 2: Stress and displacement boundary conditions

Figure 3: Equivalent stress [MPa] (left) and equivalent strain [\%] (right) for 212-grain polycrystalline aggregate with displacement boundary conditions Figure 4: Macroscopic response of the 212-grains polycrystalline aggregate

Figure 5: Scatter of macroscopic equivalent strain/stress for elasticity (left) and plasticity (right)

Figure 6: Convergence of macroscopic equivalent stresses in elasticity (left) and plasticity (right)

Figure 7: The residuum $O_{S}$ of scatter due to random crystal lattice orientations as a function of number of grain in the polycrystalline aggregates subjected to elastic deformation 


\section{FIGURES}

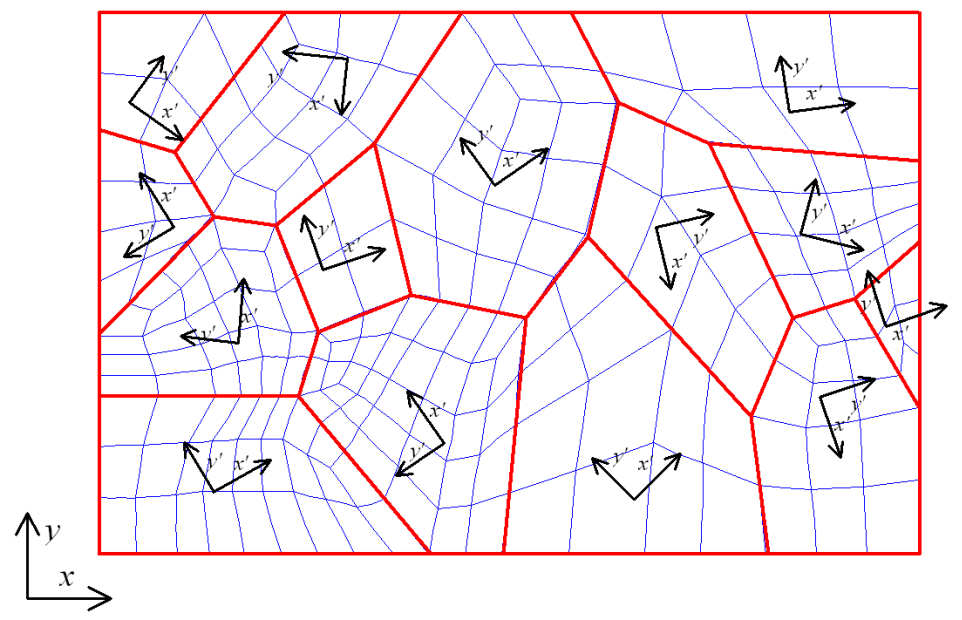

Figure 1: Voronoi tessellation with highlighted orientations of crystal lattices and finite element mesh

Stress boundary conditions

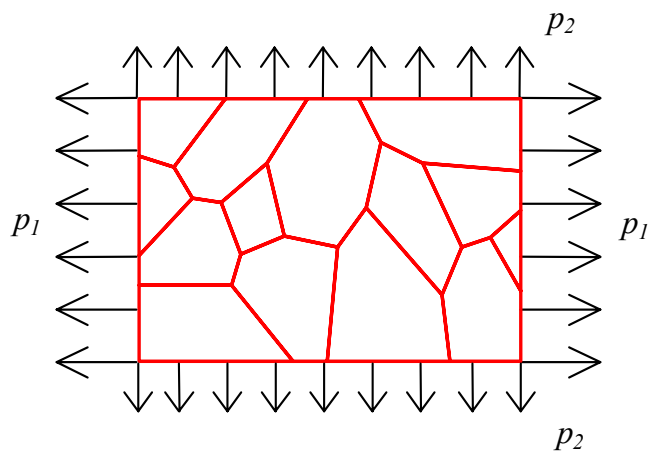

Displacement boundary conditions

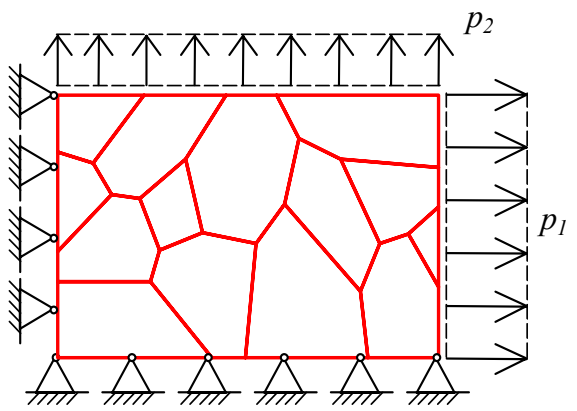

Figure 2: Stress and displacement boundary conditions 


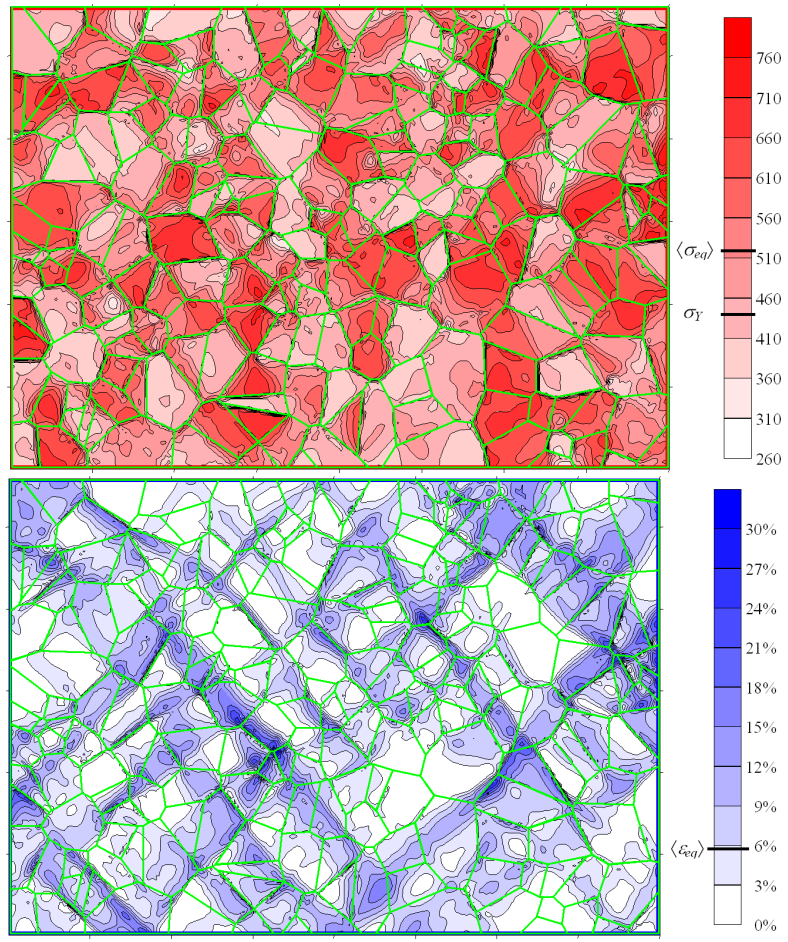

Figure 3: Equivalent stress [MPa] (left) and equivalent strain [\%] (right)

for 212-grain polycrystalline aggregate with displacement boundary conditions

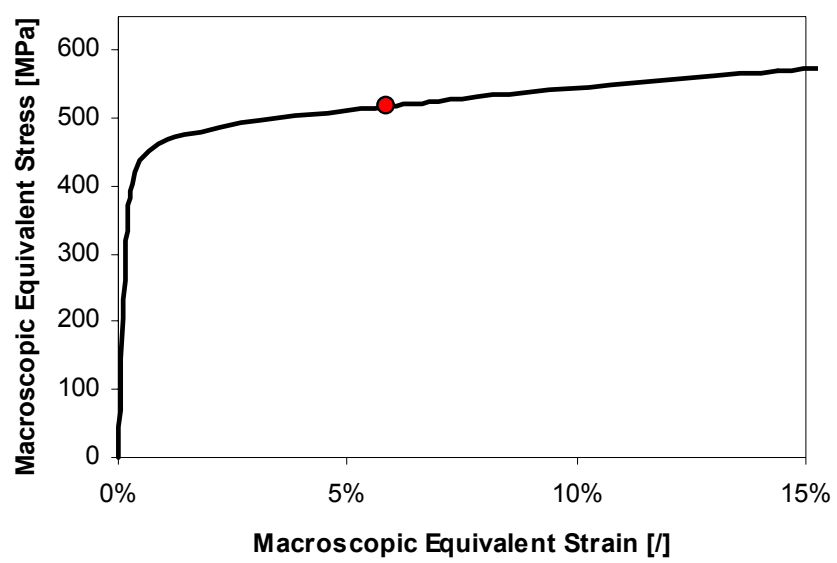

Figure 4: Macroscopic response of the 212-grains polycrystalline aggregate 

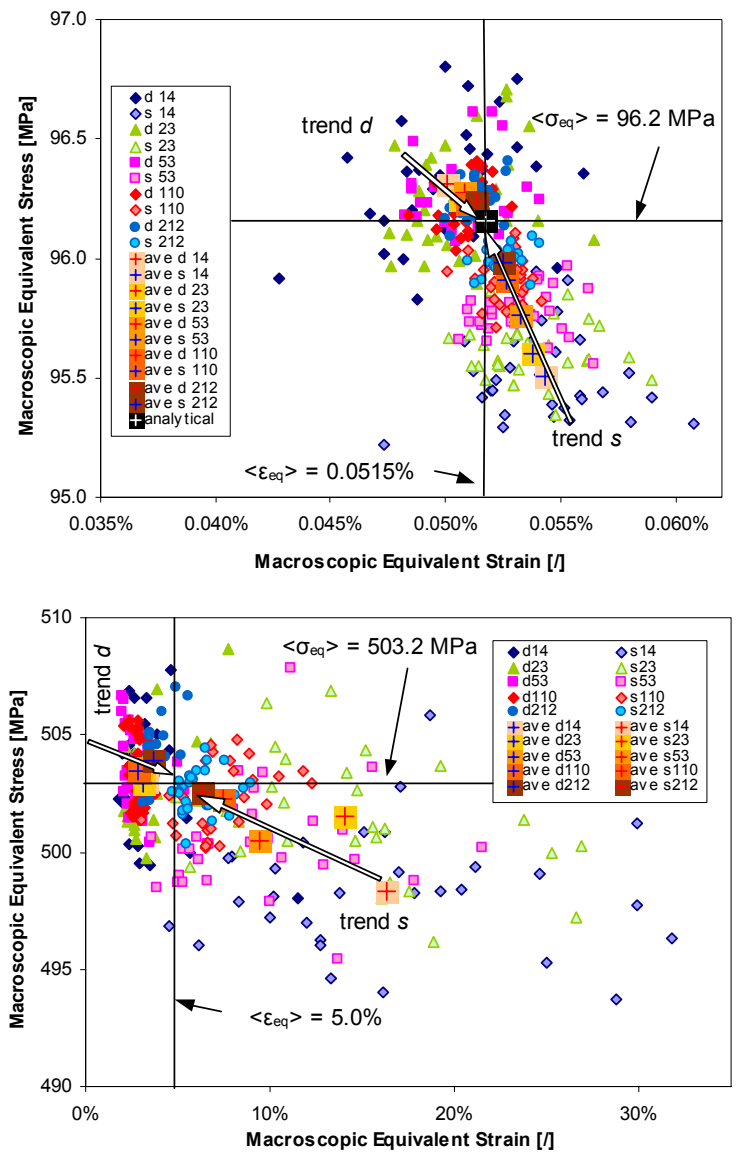

Figure 5: Scatter of macroscopic equivalent strain/stress for elasticity (left) and plasticity (right) 

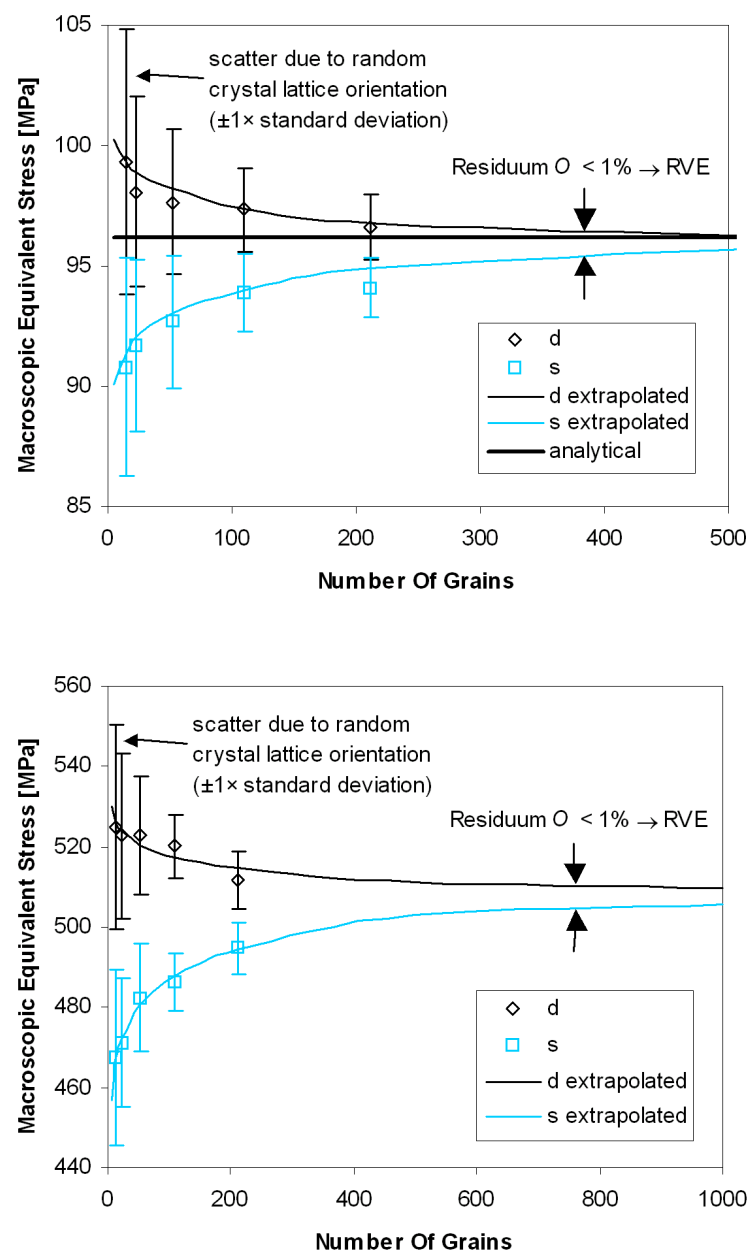

Figure 6: Convergence of macroscopic equivalent stresses in elasticity (left) and plasticity (right)

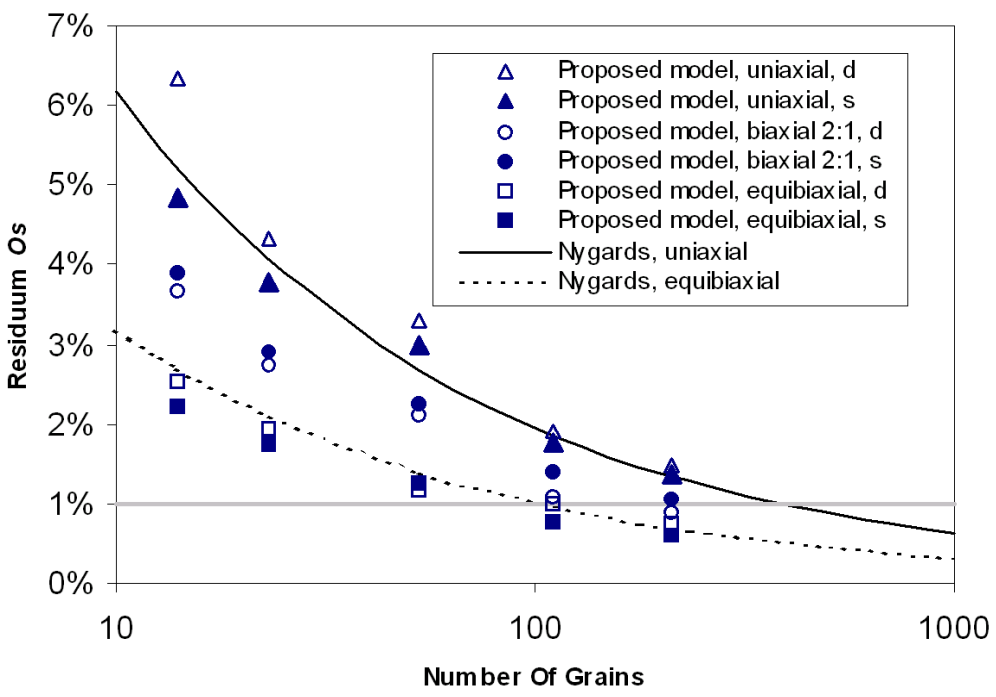


Figure 7: The residuum $O_{S}$ of scatter due to random crystal lattice orientations as a function of number of grain in the polycrystalline aggregates subjected to elastic deformation 